\title{
Local Infiltration Analgesia with Anterior Total Hip Arthroplasty under General Anaesthesia does Reduce Opioids Consumption and Pain: A Randomized, Double- Blind, Placebo-Controlled Trial Involving 106 Patients
}

\author{
Yannick NT Van Den Eeden ${ }^{1 *}$, Frank MC Van Den Eeden ${ }^{2}$, Fatih Yesilkaya ${ }^{3}$ and Bruno JG De Turck ${ }^{4}$ \\ ${ }^{1}$ Department of Orthopaedics, Krankenhaus der Barmherzigen Brüder, Nordallee 1, 54292 Trier Germany \\ ${ }^{2}$ Van den Eeden Hip Clinic, AZ Sint Elisabeth, Zottegem, Belgium \\ ${ }^{3}$ Vrije University Medical Center of Amsterdam, The Netherlands \\ ${ }^{4}$ Department of Anaesthesiology, AZ Sint Lucas, Gent, Belgium \\ *Corresponding author: Yannick NT Van Den Eeden, Department of Orthopaedics, Krankenhaus der Barmherzigen Brüder, Nordallee 1, 54292 Trier, \\ Germany
}

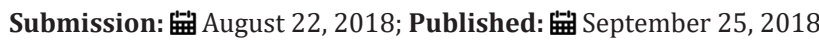

\begin{abstract}
Background: High-volume local infiltration analgesia (LIA) is widely used in total hip arthroplasty (THA) to reduce postoperative pain and opioid requirements. The efficacy of LIA in THA with different approaches to the joint remains unclear. Therefore, we examined whether intraoperative highvolume LIA in addition to a multimodal oral analgesic regimen, with direct anterior approach (DAA) for total hip arthroplasty (THA) under general anaesthesia, would further reduce acute postoperative pain and early opioid requirements consumption.
\end{abstract}

Methods: In total 106 patients were recruited. Patients scheduled for unilateral, primary DAA THA under general anaesthesia were included in this randomized, double-blind, placebo-controlled trial receiving high-volume $(150 \mathrm{ml})$ wound infiltration with ropivacaine $0.2 \%$, epinephrine $(10 \mu \mathrm{g} /$ $\mathrm{mL}$ ) and Ketorolac ( $30 \mathrm{mg}$ ) or $150 \mathrm{ml}$ saline $0.9 \%$ to evaluate the effect on early postoperative opioid consumption and on acute postoperative pain. The primary endpoint was opioid consumption $24 \mathrm{hrs}$ after surgery. The secondary endpoints were visual analogue pain-scale scores mobilized at $4 \mathrm{hrs}$ with full weight bearing and at rest at $1 \mathrm{hr}, 2 \mathrm{hrs}, 3 \mathrm{hrs}, 12 \mathrm{hrs}, 24 \mathrm{hrs}$ after surgery. The chronic analgesic consumption and surgical result at 6 weeks and 1 year after surgery were investigated.

Results: Wound infiltration with LIA did reduce postoperative opioid requirements. The average pain level $4 \mathrm{hrs}$ postoperatively mobilized was less in the LIA group. The rehabilitation progress or chronic pain after 6 weeks and 1 year showed no difference between both groups.

Conclusion: LIA was effective reducing opioid consumption and diminishing postoperative pain during mobilization using DAA THA under general anaesthesia.

\section{Introduction}

Local infiltration analgesie (LIA) in total hip arthroplasty (THA) is widely used to reduce severe postoperative pain which may delay recovery and rehabilitation $[1,2]$. The goal is to achieve adequate pain relief in combination with optimal muscle function, allowing early mobilization [3-7]. Multimodal analgesia in combination with LIA is meant to reduce the need of opiods and its side effects (nausea, vomiting, reduced gut mobility and urinary retention) to facilitate fast and safe recovery and enhance rehabilitation [8]. Since Kerr \& Kohan [2] described the LIA as an effective technique to control the acute postoperative pain following total hip and knee surgery, some studies have confirmed the efficacy of this treatment [1,9-11]. Several other studies later on reported that LIA does not provide any additional analgesic benefit or reduction in opioid consumption after THA when combined with multi modal pain strategies [12-17]. The results are conflicting and specially for less invasive surgery as the tissue sparing direct anterior THA, the role of LIA needs to be clarified. Since only limited data are available regarding LIA for THA and only one study until now is reported for THA using the DAA under spinal anaesthesia, we hypothesised that the use of LIA for DAA THA under general anaesthesia reduces direct postoperative pain and opioid consumption what enables early mobilization. We investigated the effect of LIA with direct anterior THA under general anesthesia in combination with a multimodal opioid sparing pain medication. 


\section{Methods}

In this prospective, randomized placebo-controlled trial we included all patients with osteoarthritis and avascular necrosis for elective primary direct anterior THA under general anaesthesia in a specialized outpatient hip clinic with American Society of Anesthesiologists (ASA) grades I, II and stable III. We excluded patients with

1. Rheumatoid arthritis or other inflammatory diseases,

2. Age $>85$,

3. $\mathrm{BMI}>35$

4. ASA $>3$,

5. Allergy to any of the study drugs,

6. Physical, emotional, or neurological conditions that could compromise the patient's compliance to postoperative rehabilitation and follow-up.

All the operations were performed by a single surgeon (FE) using a direct anterior Hueter approach with a positioning table (AMIS Mobile Leg positioner). In 2012 a time-based fast-track surgery protocol was started in a specialized hip clinic with a dedicated staff and a uniform approach towards giving the patients the evidence-based care principles. Exclusively the direct anterior THA is performed with implication of all the time-based care principles with implantation of identical uncemented implants for all patients. The uncemented femoral and acetabular components consisted of a Trinity cup, a MetaFix stem, a crosslinked polyethylene liner and a cobalt chrome or ceramic $32 \mathrm{~mm}$ head (Corin Group Cirencester, UK).

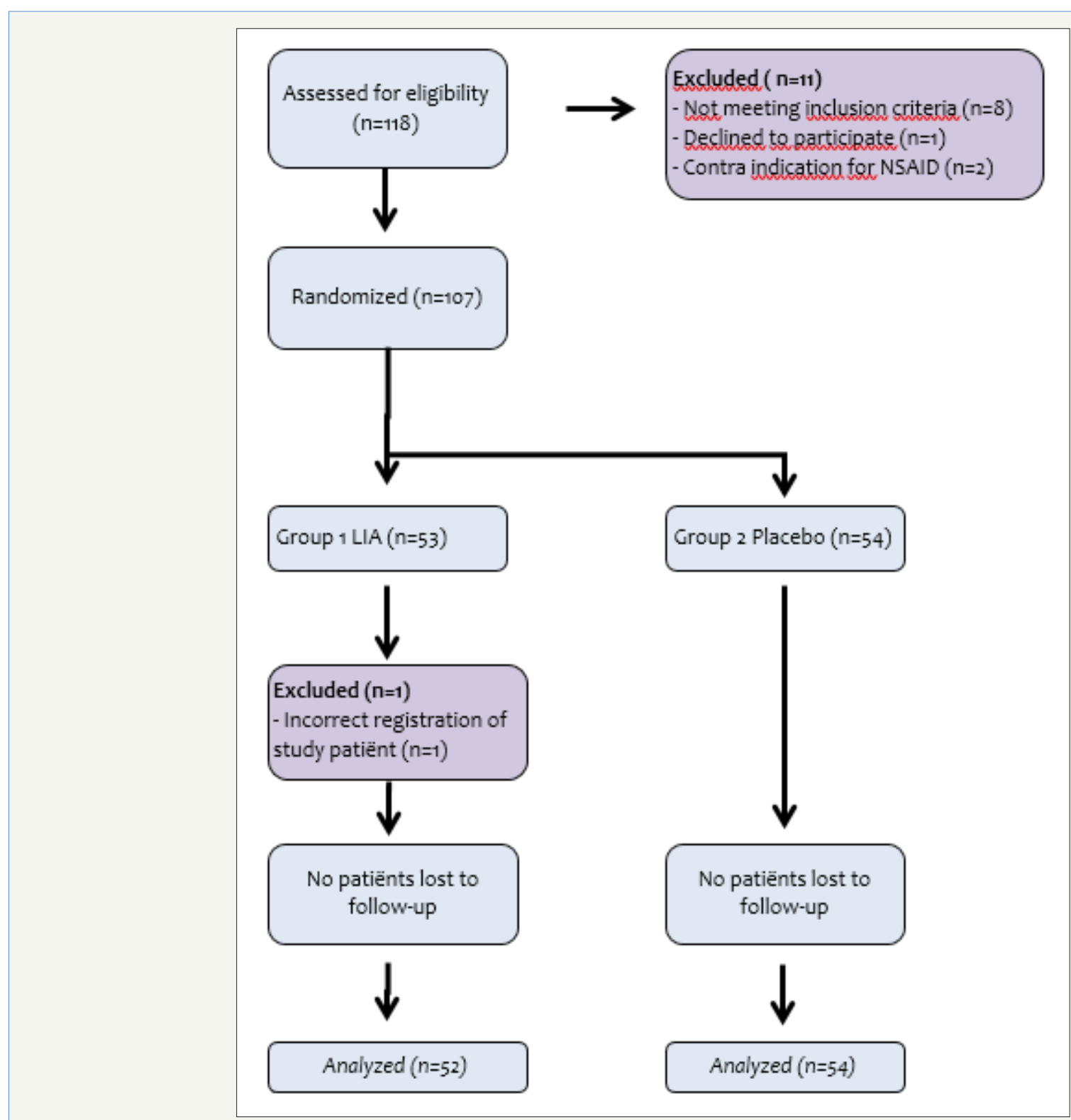

Figure 1: Flow chart of patients throughout the study. NSAID, non-steroidal anti-inflammatory drugs. 
This study was approved by the Institutional Ethical Review Board (2014.12.6.03). Patients were included after oral and written informed consent between February 15, 2016 and December 5, 2016. All the patients attended a patient educational seminar pre-operatively and were admitted on the day of surgery. 118 patients were included in the study. Figure 1 shows the flow of patients through the study. The demographic data are presented in Table 1 . The randomization and blinding into two groups were done prior to surgery. Numbered, sealed envelopes were opened by the operating nurse on the day of surgery. Group 1 patients received LIA with a total of $150 \mathrm{~mL}$ of ropivacaine $(2 \mathrm{mg} / \mathrm{mL}), 0.5 \mathrm{~mL}$ epinephrine $(1 \mathrm{mg} / \mathrm{mL})$ in a ratio of 99:1 combined with $1 \mathrm{~mL} \mathrm{Ke}-$ torolac (30mg). Group 2 patients received placebo by infiltration of $150 \mathrm{~mL}$ saline at $0,9 \%$. The anaesthetist, the surgeon, the nursing staff and the patients were all blind regarding patient allocation throughout the study. The infiltration technique of $150 \mathrm{~mL}[18,19]$ into the capsule and surrounding gluteal muscles was done after reaming the acetabulum. After introducing the femoral component, the vastus lateralis and the tensor muscle was infiltrated together with the subcutaneous tissue as described by Andersen and colleagues $[18,19]$. All patients underwent general anaesthesia using Propofol 2-4mg/kg and Sufentanyl (0.2-0.5 $\mu \mathrm{g} \mathrm{kg}$ ) associated with cisatracurium $(0.15 \mathrm{mg} \mathrm{kg}$ ). Sevoflurane (0.5-1.5MAC) inhalation anesthesia was given until wound closure. Postoperatively all patients were monitored for at least 3-4 hours in the postoperative recovery room where they are being mobilized after 4 hours. Multimodal orally administered opioid-sparing analgesia was given to all the patients: NSAIDS were given at regular intervals with IV acetaminophen 3-4xdd 1000mg, IV diclofenac 2xdd 75mg and PO Tramadol 50mg 1-4 x daily was applied. On the day of operation, the opioid rescue medication piritramide IV/IM (10 mg/ml) $2 \mathrm{mg}$ 3-4 times daily was given. Nausea was treated with intravenous ondensetron $(4 \mathrm{mg}$ ) when needed.

\section{Postoperative period}

All the patients had an identical pre- peri- and post-operative time-based fast-track procedure including a multimodal opioid sparing pain treatment regimen in combination with early fullweight bearing mobilization, starting 4 hours post-operative and short deep-vein thrombosis prophylaxis during 10 days [20,21]. Tranexamic acid (TXA) was infused as an intravenous bolus of $1,000 \mathrm{mg}$ at induction and twice $500 \mathrm{mg}$ intravenously at 4 and 6 hours post-operatively. Sufficient pain treatment with pre-operative loading (Meloxicam 15mg 2 days preoperative) was given together with a opioid sparing multimodal oral postoperative analgesic regime consisting of paracetamol 3-4 x 1,000mg in combination with Meloxicam 15mg for 14 days.

The post-operative principles of a time-based fast-track treatment were followed in which an intended discharge time after 24 hours is set, considering that the functional discharge criteria have to be fulfilled. All the patients were able to walk more than 30 meters with or without crutches, to climb stairs, to go to the toilet independently, to get into and out of bed and to dress independently apart from putting on their own socks and shoes. No restrictions in postoperative mobilization or aftercare were ordered except hip flexion of more than 120 degrees for a period of 3 months. A physiotherapy regimen post-operatively is not prescribed as a standard but used in selected cases after the first clinical control.

Nurses, who were blinded to the group in which the patient was randomized, scored the VAS for pain and the amount pain medication what was used. The extent and duration of side effects including urinary retention, cardiac arrhythmia, dizziness, hypotension, convulsions, headache and nausea and vomiting at the different times were registered. The two investigators (YE-FY) who analysed the data were blinded, as were the patients. The surgeon and the anaesthetist (FE-BT) also were blinded for both standard infiltration groups. Data were analysed after all included patients were discharged. At that moment the researchers were unblinded.

The VAS score (zero to ten, best to worst) had to be $<3$ at rest and $<5$ during mobilization. All patients were reviewed two and six weeks post-operatively. One year after surgery all the patients were interviewed for chronic pain and chronic pain medication abuse.

\section{Studied parameters}

The primary endpoint of this study was the total opioid consumption after surgery (the sum of piritramide IV/IM abuse during the 24-hour hospital stay). The secondary endpoints were postoperative pain scores evaluated by the VAS scale (zero to ten, best to worst) at mobilization at the 4th postoperative hour and at rest at the $1^{\text {st }}, 2^{\text {nd }}, 3^{\text {th }}, 12^{\text {th }}$ hour after surgery and at discharge. Patient satisfaction at 2 and 6 weeks was assessed using a numeric rating score (NRS) where 10 represented the most satisfied patient.

\section{Statistical analysis}

The sample size was calculated through simulation and based on other studies of the efficacy of LIA in THA reaching a power of over $90 \%$ with a sample size above 50 per group. The data did not fulfill a normal distribution, continuous data were therefore analyzed with Mann-Whitney U-tests. Categorical data were analyzed using Chi-squared tests or Fisher's exact test. Data are presented as frequencies, means $( \pm S D)$ and ranges in different tables. The level of significance was chosen to be 0.05 . Data analysis was conducted with IBM-SPSS Statistics version 24 (IBM-SPSS, Armonk, New York).

\section{Results}

Between 15 February 2016 and 5 December 2016, a total of 118 patients were assessed for eligibility; 8 patients did not met the inclusion criteria, 1 declined participation and 2 patients had a contra indication for further use of NSAIDS. 107 patients were included in the study, randomized into two groups of 53 and 54. In group I (LIA) 1 patient had incomplete registration of their painscore leaving 52 patients in the LIA group and 54 in the placebo group. All the data for primary endpoint were registered for all the remaining patients. Baseline demographics showed similar values in both groups except the BMI (P-value: 0.03) Table 1). 
Table 1: Patient demographics. Data are presented as mean and range. unless otherwise stated. BMI. body mass index. ASA: American Society of Anesthesiologists; HOOS: Hip-disability and Osteoarthritis Outcome Score.

\begin{tabular}{|c|c|c|c|}
\hline & Group 1 LIA (n=52) & Group 2 Placebo(n=54) & p-value \\
\hline Gender: M/F & $24 / 28$ & $23 / 31$ & 0.09 \\
\hline Age. years (range) & $67.5(49-80)$ & $63.7(44-81)$ & 0.03 \\
\hline BMI kg/m²(range) & $26.4(19.6-34)$ & $24.9(17.6-33.9)$ & 0.08 \\
\hline ASA score I & 27 & 34 & 0.06 \\
\hline ASA score II & 25 & 1 & 0.46 \\
\hline Living situation (n) Alone & 2 & $60(40-90)$ & 0.44 \\
\hline Duration of surgery. min & $62(45-115)$ & $322(100-650)$ & 0.07 \\
\hline Blood loss. ML & $951(100-950)$ & $91.7(36-155)$ & \\
\hline HOoS pre-operative(n=44) & & & \\
\hline
\end{tabular}

Mean duration of surgery was $60 \mathrm{~min}(45-115)$ with an average blood-loss of 340mL (100-950) and a mean length of hospital stay (LOS) of 26 hours (range 22-28 hours) did not differ significantly between the two groups. The mean and range scores of the visual analog scale (VAS) is shown in Table 2, at four hours mobilized and at one, two and three, twelve hours in rest post-operatively. After mobilization 4 hours postoperative a significant difference is shown (P-value: 0,03). No differences at rest were analyzed (Table 3).

Table 2: Mean Visual analog scale (VAS) at four hours mobilized and at one, two and three hours post-operatively, in rest and all the other times registered for the two groups. Data are presented as mean and range, unless otherwise described.

\begin{tabular}{|c|c|c|c|}
\hline & LIA-Group (N=52) & Placebo Group (N=54) & p-value \\
\hline 4h (mobilized) & $2.85(1-5)$ & $3.31(1-7)$ & 0.03 \\
\hline 1h & $4.21(2-7)$ & $4.57(1-8)$ & 0.32 \\
\hline 2h & $3.32(0-8)$ & $3.78(0-7)$ & 0.08 \\
\hline 3h & $2.84(0-5)$ & $3.04(0-5)$ & 0.40 \\
\hline morning day 1 post-op & $2.41(0-5)$ & $2.20(0-6)$ & 0.16 \\
\hline At discharge & $2.48(0-4)$ & $2.31(0-6)$ & 0.43 \\
\hline At intake & $7.41(4-10)$ & $7.20(3-10)$ & 0.11 \\
\hline
\end{tabular}

Table 3: Numbers of patients using (rescue) pain medication for the two groups. Data analysis was performed with a chi-squared test.

\begin{tabular}{|c|c|c|c|c|}
\hline \multicolumn{2}{|c|}{ Pain Medication } & Gr I & Gr II & p-value \\
\hline \multirow{3}{*}{ Day 0 } & $1 \mathrm{hr}$ & 54 & 52 & 0.99 \\
\cline { 2 - 5 } & $2-4 \mathrm{hrs}$ & 52 & 51 & 0.97 \\
\cline { 2 - 5 } & $8 \mathrm{hrs}$ & 51 & 50 & 0.96 \\
\hline Day I & Morning & 51 & 50 & 0.96 \\
\hline \multicolumn{2}{|c|}{ Rescue Medication (Dipidolor) } & $1 \mathrm{hr}$ & 9 & 0.04 \\
\hline \multirow{2}{*}{ Day 0 } & $2-4 \mathrm{hrs}$ & 19 & 2 & 0.01 \\
\hline & $8 \mathrm{hrs}$ & 0 & 0 & \\
\hline Day I & Morning & 0 & 0 & \\
\hline
\end{tabular}

The cumulative consumption of intravenous or intramuscular piritramide direct post-operatively was $136 \mathrm{mg}$ in the placebo group with a mean consumption of 2,52 (0-6)mg in comparison with a total consumption of $60 \mathrm{mg}$ in the LIA group with a mean consumption of $1,15(0-4$.)mg. The opioid rescue drug was given to 21 patients out of 54 in the placebo group and 9 out of 52 in the LIA group (p-value: 0,02). The cumulative consumption of Tramadol during hospital stay was respectively 5000mg (mean 92,6mg) in the placebo group and $4200 \mathrm{mg}$ (mean $80,8 \mathrm{mg}$ ) in the LIA group without statistical significance. The total of 39 out of 54 patients in 
the placebo group were using Tramadol and 33 out of 52 patients in the LIA group (Table 4). The use of Tramadol at home was low and was only prescribed if required; 3 times in the placebo group and 2 times in the LIA group.

Table 4: The total and average usage of Piritramide and Tramadol in mg over the first $24 \mathrm{~h}$ postoperatively in the two groups and the amount $(\mathrm{N})$ of patients using it.

\begin{tabular}{|c|c|c|c|}
\hline & LIA-Group (N=52) & Placebo Group (N=54) & p-Value \\
\hline Piritramide: Total & $60 \mathrm{mg} / 52$ & $136 \mathrm{mg} / 54$ & 0.02 \\
\hline Average: & $1.15 \mathrm{mg}$ & $2.52 \mathrm{mg}$ & 0.03 \\
\hline $\mathrm{N}=$ & $9 / 52$ & $21 / 54$ & 0.43 \\
\hline Tramadol: Total: & $4200 \mathrm{mg} / 52$ & $5000 \mathrm{mg} / 54$ & \\
\hline Average: & $80.8 \mathrm{mg}$ & $92.6 \mathrm{~m}$ & 0.45 \\
\hline $\mathrm{N}=$ & $33 / 52$ & $39 / 54$ & \\
\hline
\end{tabular}

All the side effects recorded (itching, nausea and vomiting) were similar in both groups with a total of $14 \%$ of all patients with no differences between both groups. During the $24 \mathrm{~h}$ study period no adverse events were reported. Postoperative pain intensity scores and analgesic consumption at 2 weeks in rest and during walking showed no difference between both groups. There was 1 surgical complication in the placebo group. The patient was re-admitted and successfully re-operated with exchange of the hip prosthesis, due to dislocation after stem subsidence. 1 superficial insufficient wound healing was seen which was treated conservatively. No signs of deep venous thrombosis or any other non-surgical complication was found.

\section{Discussion}

The data concerning the effect of the infiltration of local anaesthetic in THA are limited, unclear and conflicting [22]. LIA in total knee arthroplasty (TKA) seems to be effective and is in general recommended being cheap and easy to perform [11,23]. A single peri-articular infiltration in TKA reduced postoperative pain and opioid consumption what was most apparent during the first $6 \mathrm{~h}$ in patients. In analogy to this outcome we would expect some kind of effect of LIA in the tissue sparing anterior THA in the first hours postoperatively, especially after general anaesthesia.

In this randomized, placebo-controlled trial we investigated the effect of local infiltration anesthesia (LIA) in combination with the direct anterior approach (DAA) for THA under general anesthesia. We found that LIA does provide an analgesic effect after DAA THA in addition to a multimodal analgesic regimen. We found clear statistically differences between both groups in opioid consumption during the first 24 hours postoperatively. The consumption of piritramide in the placebo group was double as high as in the LIA group. An equal correlation with the patient registered average pain scores at rest could not be adjusted. After the first postoperative mobilization at 3 to 4 hours, a statistical difference between the LIA group and the placebo group could be registered ( $p$ value: 0.03 ). Less pain does facilitate first mobilization and rehabilitation in combination with less rescue pain medication in the early postoperative phase. In our study we did not use a glucocorticoid pre-operatively to make our results comparable with the other studies concerning the effect of LIA. We chose to evaluate acute post-surgical pain by the VAS at rest and at mobilization. The primary and secondary endpoint of our study was during a total time period of 24 hours. In our timebased fast-track setting of 24 hours, the VAS is the most well-known and simplest method for acute pain evaluation after THA and TKA.

Concerning the effect and role of the infiltration of local anesthetic for THA the data are limited and contradictory. In Kerr \& Kohan [2] reported satisfactory results for pain control after THA without the use of a control group. The same good response with LIA was recorded in two other studies in comparison with a placebo group $[1,10]$. In the first study a combination of the results after THA and TKA were registered and in the second study no multimodal pain scheme was used peri-operatively. To make the outcome of our study comparable with other studies, we used the same LIA mixture with or without Ketorolac, as described by Andersen and others, knowing that the pain-effect of Ketorolac as a supplement NSAID could influence the outcome mutually of the VAS evaluation as described in other studies [13,23,24].

The role of LIA in total hip replacement needs to be clarified. Some studies have reported superior outcome with LIA regarding postoperative analgesia, opioid consumption [2,10,25-28] and shortening of the hospital stay [15,24]. Andersen et al. [10] showed a $62 \%$ reduction in opioids consumption during the first postoperative day. Other studies reported no additional benefit in analgesic effect or reduction of opioid consumption after a single dose of $150 \mathrm{~mL}, 0.2 \%$ ropivacaine with epinephrine following THA [8,1417] Several trials investigating the effect of LIA following THA have used different pain treatment modalities, and different approaches to the hip joint $[13,14,17,24]$. Postoperative administration of LIA to the wound may be effective in many operative procedures [29] but concerning THA, procedure-specific studies are needed to solve the role of LIA [23].

In our study, all patients received general anaesthesia and a standardized anterior approach to the hip joint. All patients were treated under general anaesthesia using a uniform anaesthetic regimen. We expected the influence of local anaesthetic infiltration to be greatest at the first postoperative hours after terminating general anaesthesia $[10,11,18,19]$. Excessive pain is to be expected mostly in the early postoperative period during the first 3 to 4 hours. The VAS after surgery ranged from 0 to 9 at various times, but only af- 
ter mobilization at 4 hours postoperative, a statistical difference in pain scores between both groups was registered in favour of the LIA group. Despite the BMI being statistically higher in the LIA group a correlation between longer operating time and more tissue damaging resulting in increased pain could not be made. Furthermore, the total consumption of opioids after the operation did differ in the early postoperative phase. In the LIA group the total consumption was $60 \mathrm{mg}$ out of 52 patients against $136 \mathrm{mg}$ out of 54 patients for the placebo group. The total abuse of opioids was statistically lower during the first 4 postoperative hours in the group applying LIA. In contrast with this outcome, the consumption of tramadol was practically equal in both groups during the first postoperative day being first choice in pain treatment in combination with the described multi modal pain regime.

Several studies using a different approach to the hip joint did not show a beneficial analgesic effect of infiltration in THA $[8,15,17]$. Our trial is the second one to evaluate the effect of LIA using the anterior approach for THA. In the only other study concerning the effectivity of LIA following the anterior approach THA, all patients received spinal anaesthesia with bupivacaine and propofol $(1-2 \mathrm{mcg} / \mathrm{ml})$ in combination with a different postoperative standardized pain regimen with acetaminophen $(4 \mathrm{x} 1 \mathrm{~g})$, celecoxib $200 \mathrm{mg}$, gabapentin $300 \mathrm{mg}$ and tramadol 100mg [13]. In this study no differences in pain scores after 4 hours were detected and the use of (rescue) pain medication did not differ between the two groups. If the prolonged anaesthetic effect, due to spinal anaesthesia with bupivacaine, plays a role in pain reduction the first postoperative hours, remains unclear, but a prolonged analgesic effect after neuraxial anaesthesia could be expected knowing the long acting anaesthesia of bupivacaine (Marcaine) with a half-life of around 3 hrs in adults.

In our study the average degree of pain intensity was significantly lower after the first mobilization 4 hrs postoperative. The lesser pain intensity facilitates the LIA patients to walk better with full weight bearing with or without crutches and enables to dress independently and get in and out of the bed much easier. At all other times at rest at $1 \mathrm{hr}, 2 \mathrm{hrs}$ and $3 \mathrm{hrs}$ the VAS painscore was slightly lower in the LIA group without statistical significance. At all the later pain recordings a difference between the two groups was lacking. Furthermore, there was no difference in operating time, LOS, blood loss or side effects except the LIA patients walk and revalidate with more comfort and less pain.

All our patients received a pain regime at home including acetaminophen (paracetamol) 3-4 X 1000mg and meloxicam 15mg once daily as a part of a postoperative multimodal pain regimen. Tramadol at home was prescribed only if required, 3 times in the placebo group and 2 times in the LIA group. The average VAS score at 2 and 6 weeks postoperative showed no difference between the two groups. Only one patient in the LIA group scored a VAS over 7 dues to hypersensibility of the lateral femoral cutaneal nerve (LFCN). This kind of pain sensation was only seen once in the total group of patients. All patients were directly discharged home on the first postoperative day.
A recent systematic review of Johnson et al. 2016 showed no statistically significant differences between neuraxial and general anaesthesia for mortality, surgical duration, surgical site or chest infections, nerve palsies, postoperative nausea and vomiting, or thromboembolic disease when antithrombotic prophylaxis was used. Although spinal or epidural anaesthesia is concerned to be the golden standard for total hip replacement, the grade of relaxation of the musculature around the hip joint seems to be more profound under general anaesthesia [30]. This effect could reduce operative tissue damage at the surgical site associated with lesser pain, swelling and hematoma [31]. In the study of Den Hartog [5] using LIA with patients undergoing a DAA THA receiving spinal anaesthesia no clinically relevant effect was seen 4 hours after surgery, in contrast with our study group of patients receiving general anaesthesia where an early reduction of postoperative consumption of opioids after LIA could be adjusted [13].

We hypothesized that a single-shot of local infiltration in combination with a multimodal pain treatment would reduce postoperative pain scores after first mobilization and would reduce opioid abuse. Knowing that the advantages of the anterior approach, regarding early mobilization and rehabilitation, diminishing opioids abuse in combination with lesser pain does allow early discharge under safe conditions with very little side effects.

In conclusion, in this randomized study we could determine that the use of a single dose perioperative infiltration of local anesthetic (LIA) in direct anterior approach (DAA) THA under general anaesthesia, in combination with a multimodal pain regime, does lower the consumption of opioids in the first 24 hours postoperatively and secures early mobilization and rehabilitation with less pain. Based on the results we do recommend the use of LIA in direct anterior muscle sparing THA being cheap and easy to use, especially in patients receiving general anesthesia.

\section{Acknowledgement}

FE and YE contributed to planning of the study and drafting of the article. YE, BT and FY conducted the data analyses and interpreted the results. Special thanks to the nursing staff who participated in collection of the registry data and Margaretha Emmerechts, the operating nurse, being responsible for organizing the study intervention. Thanks to the Institute for Orthopaedic Research and Training (IORT), University Hospital Pellenberg Leuven, Belgium who carried out the statistical analysis No competing interests declared.

\section{References}

1. Bianconi M, Ferraro L, Traina GC, Zanoli G, Antonelli T, et al. (2003) Pharmacokinetics and efficacy of ropivacaine continuous wound instillation after joint replacement surgery. Br J Anaesth 91(6): 830-835.

2. Kerr DR, Kohan L (2008) Local infiltration analgesia: a technique for the control of acute postoperative pain following knee and hip surgery: a case study of 325 patients. Acta Orthop 79(2): 174-183.

3. Eeden van den YNT, De Turck BJG, Eeden van den FMC (2017) 24 hours stay after hip replacement. Acta Orthopaedica 88(1): 24-28. 
4. Glassou EN, Pedersen AB, Hansen TB (2014) Risk of re-admission, reoperation, and mortality within 90 days of total hip and knee arthroplasty in fast-track departments in Denmark from 2005 to 2011. Acta Orthop 85(5): 493-500.

5. Hartog den YM, Mathijssen NM, Vehmeijer SB (2013) Reduced length of hospital stay after the introduction of a rapid recovery protocol for primary THA procedures. Acta Orthop 84(5): 444-447.

6. Husted H, Hansen HC, Holm G, Bach-Dal C, Rud K, et al. (2010) What determines length of stay after total hip and knee arthroplasty? A nationwide study in Denmark. Arch Orthop Trauma Surg 130(2): 263268.

7. Husted H, Jensen CM, Solgaard S, Kehlet H (2012) Reduced length of stay following hip and knee arthroplasty in Denmark 2000-2009: from research to implementation. Arch Orthop Trauma Surg 132(1): 101-104.

8. Andersen KV, Pfeiffer-Jensen M, Haraldsted V, Søballe K (2007) Reduced hospital stay and narcotic consumption, and improved mobilization with local and intraarticular infiltration after hip arthroplasty: a randomized clinical trial of an intraarticular technique versus epidural infusion in 80 patients. Acta Orthop 78(2): 180-186.

9. Affas F, Nygårds EB, Stiller CO, Wretenberg P, Olofsson C (2011) Pain control after total knee arthroplasty: a randomized trial comparing local infiltration anesthesia and continuous femoral block. Acta Orthop 82(4): 441-447.

10. Andersen J, Poulsen T, Krogh B, Nielsen T (2007) Postoperative analgesia in total hip arthroplasty: a randomized, double-blind, placebo-controlled study on peroperative and postoperative ropivacaine, ketorolac and adrenaline wound infiltration. Acta Orthop 78(2): 187-192.

11. Niemeläinen M, Kalliovalkama J, Aho AJ, Moilanen T, Eskelinen A (2014) Single periarticular local infiltration analgesia reduces opiate consumption until 48 hours after total knee arthroplasty. A randomized placebo-controlled trial involving 56 patients. Acta Orthop 85(6): 614619.

12. Dobie I, Bennett D, Spence DJ, Murray JM, Beverland DE (2012) Periarticular local anesthesia does not improve pain or mobility after THA. Clin Orthop Relat Res 470(7): 1958-1965.

13. den Hartog YM, Mathijssen NM, van Dasselaar NT, Langendijk PN, Vehmeijer SB (2015) No effect of the infiltration of local anaesthetic for total hip arthroplasty using an anterior approach: a randomised placebo-controlled trial. Bone Joint J 97-B(6): 734-740.

14. Hofstad JK, Winther SB, Rian T, Foss OA, Husby OS, et al. (2015) Perioperative local infiltration anesthesia with ropivacaine has no effect on postoperative pain after total hip arthroplasty. Acta Orthop 86(6): 654-658.

15. Lunn TH, Husted H, Solgaard S, Kristensen BB, Otte KS, et al. (2011) Intraoperative local infiltration analgesia for early analgesia after total hip arthroplasty: a randomized, double-blind, placebo-controlled trial. Reg Anesth Pain Med 36(5): 424-429.

16. Solovyova O, Lewis CG, Abrams JH, Benson GJ, Joyce ME, et al. (2013) Local infiltration analgesia followed by continuous infusion of local anesthetic solution for total hip arthroplasty: a prospective, randomized, double-blind, placebo-controlled study. J Bone Joint Surg Am 95(21): 1935-1941.

17. Zoric L, Cuvillon P, Alonso S, Demattei C, Vialles N, et al. (2014) Singleshot intraoperative local anaesthetic infiltration does not reduce morphine consumption after total hip arthroplasty: a double-blinded placebo-controlled randomized study. Br J Anaesth 112(4): 722-728.

18. Andersen LØ, Husted H, Otte KS, Kristensen BB, Kehlet H (2008) Highvolume infiltration analgesia in total knee arthroplasty: a randomized, double-blind, placebo-controlled trial. Acta Anaesthesiol Scand 52(10): 1331-1335.

19. Andersen LØ, Larsen GL, Kristensen BB, Husted H, Otte KS, et al. (2010) Analgesic efficacy of local anaesthetic wound administration in knee arthroplasty: volume vs concentration. Anaesthesia 65(10): 984-990.

20. Husted H, Lunn TH, Troelsen A, Larsen GL, Kristensen BB, et al. (2011) Why still in hospital after fast-track hip and knee arthroplasty? Acta Orthop 82(6): 679-684.

21. Jorgensen CC, Kehlet H, Lundbeck Foundation Centre for Fast-track H and Knee replacement collaborative G (2013) Role of patient chararteristics for fast-track hip and knee arthroplasty. Br J Anaesth 110(6): 972-980.

22. Eeden van den FMC, Eeden van den YNT (2018) Local infiltration anesthesia in total knee and total hip arthroplasty: A brief review. J Anest \& Inten Care Med 5(3): 1-3.

23. Andersen LØ, Otte KS, Husted H, Larsen LG, Kristensen B, et al. (2011) High-volume infiltration analgesia in bilateral hip arthroplasty A randomized, double-blind placebo-controlled trial. Acta Orthop 82(4): 423-426.

24. Andersen KV, Nikolajsen L, Daugaard H, Andersen NT, Haraldsted V, et al. (2015) Local infiltration analgesia is not improved by postoperative intra-articular bolus injections for pain after total hip arthroplasty. A randomized, double-blind, placebo-controlled study with 80 patients. Acta Orthop 86(6): 647-653.

25. Kuchálik J, Granath B, Ljunggren A, Magnuson A, Lundin A, et al. (2013) Postoperative pain relief after total hip arthroplasty: a randomized, double-blind comparison between intrathecal morphine and local infiltration analgesia. Br J Anaesth 111(5): 793-799.

26. Murphy TP, Byrne DP, Curtin P, Baker JF, Mulhall KJ (2012) Can a periarticular levobupivacaine injection reduce postoperative opiate consumption during primary hip arthroplasty? Clin Orthop Relat Res 470(4): 1151-1157.

27. Gupta T, Garg N, Gupta M (2014) Local infiltration analgesia following total hip replacement: a review of current literature. Chinese Journal of Traumatology 17(5): 293-297.

28. Liu W, Cong R, Li X, Wu Y, Wu H (2011) Reduced opioid consumption and improved early rehabilitation with local and intraarticular cocktail analgesic injection in total hip arthroplasty: a randomized controlled clinical trial. Pain Med 12(3): 387-393.

29. Johnson RL, Kopp SL, Burkle CM, Duncan CM, Jacob AK, et al. (2016) Neuraxial vs general anaesthesia for total hip and total knee arthroplasty. a systematic review of comparative-effectiveness research. Br J Anaesth 116(2): 163-176

30. Bush CA, Whitehouse MR, Shore BJ, MacDonald SJ, McCalden RW, et al. (2010) The efficacy of periarticular multimodal drug infiltration in total hip arthroplasty. Clin Orthop Relat Res 468(8): 2152-2159.

31. Scott CE, Streit J, Biant LC, Breusch SJ (2012) Periarticular infiltration in total hip replacement: effect on heterotopic ossification, analgesic requirements and outcome. Arch Orthop Trauma Surg 132(5): 703-709. 
Creative Commons Attribution 4.0 International License

For possible submissions Click Here

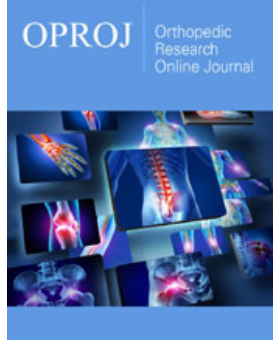

Orthopedic Research Online Journal

\section{Benefits of Publishing with us}

- High-level peer review and editorial services

- Freely accessible online immediately upon publication

- Authors retain the copyright to their work

- Licensing it under a Creative Commons license

- Visibility through different online platforms 